\title{
Experiential training during COVID-19 pandemic: a virtual attachment experience from a college of pharmaceutical sciences in the United Arab Emirates
}

\author{
Syed Arman Rabbani iD, Tarun Wadhwa, Sathvik B Sridhar, Javedh Shareef, Areeg Anwer Ali, Padma GM Rao \\ Department of Clinical Pharmacy and Pharmacology, RAK Medical and Health Sciences University, United Arab Emirates
}

Keywords

COVID-19

Experiential training

Online

Pharmacy

United Arab Emirates

Virtual

\section{Correspondence}

Dr Syed Arman Rabbani

Associate Professor

Department of Clinical Pharmacy and Pharmacology

RAK College of Pharmaceutical Sciences

RAK Medical and Health Sciences University

United Arab Emirates

arman@rakmhsu.ac.ae

\begin{abstract}
Description: The COVID-19 pandemic compelled the traditional higher educational institutions to make a quick transition to the virtual instruction model. Considering the COVID-19 restrictions, the Department of Clinical Pharmacy and Pharmacology at Ras Al Khaimah College of Pharmaceutical Sciences, United Arab Emirates restructured their original onsite experiential training for final year Bachelor of Pharmacy students as a virtual online experience to ensure educational continuity. Evaluation: The virtual experiential training was conducted over two weeks and was imparted employing different virtual platforms like Google Classroom, Google Meet, Google Forms, Google Docs, etc. As a part of the community pharmacy training, the students were given different e-activities and tasks like e-case scenario analysis and interpretation, e-prescription screening, virtual patient education and were shown various 3-D animated videos related to community pharmacy. For the hospital training, the students were given different e-cases for developing pharmaceutical care plans, e-case scenario analysis, e-drug information query and were shown different animated videos related to rounds of different hospital departments. Conclusion: The virtual experiential training was successfully conducted achieving the desired learning outcomes and was well received by the students. Based on this experience, it is recommended that efforts should be made to develop an integrated model for experiential training in the future, which can be an amalgamation of online tools and traditional experiential methods.
\end{abstract}

\section{Background and context}

Experiential training is a critical component of the medical and health sciences curricula (Kallail, Shaw, Hughes, \& Berardo, 2020), which helps the students gain confidence in their abilities and be active and useful healthcare team members. With experiential training, the students learn to apply their classroom knowledge to real-life hospital and community settings (Rathbun et al., 2012). It assists future healthcare providers in developing and refining the skills necessary to provide comprehensive pharmaceutical care (Boyce et al., 2020). Practice experiences, community and hospital, extend the practice exposure of students and permit them to experience direct responsibilities of patient care in different outpatient and inpatient settings (Akel et al., 2020). The dual practice-based experiences involving both community pharmacy and hospital settings provide the students with an appropriate landscape to develop their competence and become proficient patient-centered pharmacists (Rathbone et al., 2019).

The COVID-19 pandemic has made a significant impact worldwide, compelling traditional higher education institutions to quickly transition to the virtual 
instruction model (Christian, McCarty, \& Brown, 2020). At the peak of the COVID-19 pandemic, restrictions were imposed on all higher educational institutions and associated teaching hospitals, forcing the educators to look for alternatives to maintain academic continuity, mainly experiential training (Elnaem, Nazar, \& Rahman, 2020; Hussain, Al-Mannai, \& Agouni, 2020; Wahab \& Zainal, 2020). Keeping in view the challenging situation, the Department of Clinical Pharmacy and Pharmacology at Ras Al Khaimah College of Pharmaceutical Sciences restructured their experiential training, making a quick and seamless transition from the original onsite to the online mode of training without compromising the quality. This short communication highlights the Department's experience of experiential training during the peak of the COVID-19 pandemic.

\section{Educational Description}

\section{Experiential training pre-COVID-19 pandemic}

The original experiential training for final year students at the College of Pharmaceutical Sciences involves community pharmacy and hospital placements. Both the placements are carried out in the final year of the Bachelor of Pharmacy programme. Community pharmacy training involves posting the students to the different primary health care centres under the Ministry of Health and Prevention and private community pharmacies for learning skills and acquiring the required competencies under the guidance of qualified, experienced and licensed preceptors along with the course faculty. The training comprises specific activities related to student introduction to community pharmacy setting and workflow, applying pharmaceutical skills in a community pharmacy setting, pharmaceutical products, and related professional activities. Hospital rotations involve posting students in the different departments such as internal medicine, cardiology, neurology, nephrology, psychiatry, nutrition, and hospital pharmacy of Ministry of Health and Prevention approved teaching hospitals. During the rotations, the students gain knowledge and skills from clinicians and pharmacists about common disease conditions and their management with drugs, clinical data, and interpretation. Students also learn about patient counselling, drug information services, and adverse drug reactions reporting and monitoring.

\section{Experiential training during COVID-19 pandemic}

Considering the importance of practice-based education, the ongoing COVID-19 situation, and the Commission of Academic Accreditation recommendations, the original experiential training comprising of community pharmacy and hospital placements was reorganised. Experiential training was converted to virtual training without compromising the students' learning and quality of training content in challenging times. This virtual experiential training was conducted for over two weeks with faculty supervisors as the students' preceptors. A total of 52 students and seven preceptors were a part of this virtual experiential training.

The training was imparted employing different virtual platforms like Google Classroom, Google Meet, Google Forms, Google Docs, etc. Google Classroom was used for uploading all the training-related activities, including virtual videos, e-case scenarios, and other material. Google Meet was used for having virtual discussions among the student groups and the preceptors and for case presentations. Google Forms and Docs were used for case-based assessments and for submitting different e-activities to the preceptors.

The students documented all their activities during virtual experiential training in a specially made e-daily reflective diary. Each day after completing all the virtual activities, the students had a one-to-one discussion with their respective preceptors on Google Meet. After each of the virtual placements, the students gave end rotation presentations and submitted final rotation reports. All the virtual activities, case presentations, rotation reports and e-daily reflective diaries of the students were assessed using standard rubric forms by the preceptors. Feedback was taken from the students at the end of the virtual experiential training experience by sending out Google forms.

\section{Virtual community pharmacy placements}

During the virtual community pharmacy rotation, the students were given a virtual tour of a model community pharmacy with 3-D animated videos. Students were provided with reading materials covering different aspects of community pharmacy, such as policies and laws governing the functioning of a community pharmacy, types of healthcare-related services provided by community pharmacists, and procedures for handling expired/near expiry medications. Students were also shown different virtual videos on various functional areas of community pharmacy like dispensing over the counter (OTC) medications, prescription only, pharmacist only medications, medical equipment, and cosmetics. Furthermore, students were given virtual tasks like contacting the nearest community pharmacy and identifying different medical equipment and cosmetic products sold, identifying different products used to 
treat common conditions like cold and cough, constipation, acne, hair loss, etc.

More importantly, students were trained in virtual patient education with Google Meet's help, where they did one-on-one medication counselling for simulated patients. Students were given different e-case scenarios to interpret and analyse drug-related problems and symptom assessment and management. They were also trained in e-prescription screening, where different e-prescriptions were given to analyse for the legality, legibility, ambiguity, and drug-related problems. Figure 1 depicts all the virtual community pharmacy placement activities employed during the COVID-19 pandemic.

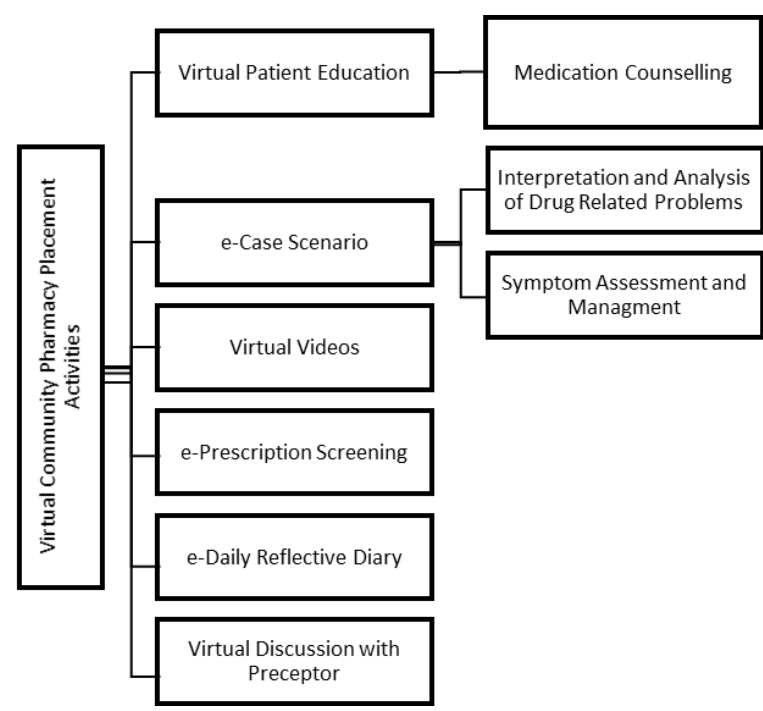

Figure 1: Virtual community pharmacy training experience

\section{Virtual hospital placements}

Virtual hospital rotation covered all the core areas of hospital practice, such as internal medicine, cardiology, neurology, nephrology, psychiatry, nutrition, hospital pharmacy, and drug information. The students were given one case scenario for writing a pharmaceutical care plan and one case scenario for analysis and interpretation for each of the core areas. The case scenarios covered different diseases like community-acquired pneumonia, acute bacterial gastroenteritis, myocardial infarction, heart failure, acute kidney injury, end-stage renal disease, stroke, schizophrenia, and bipolar disorder. After analysing the case scenarios for pharmaceutical care issues, the students had virtual case discussions with the preceptor using Google Meet.

In addition to this, students were shown hospital pharmacy related virtual videos explaining the overview of hospital pharmacy, different hospital pharmacy services, roles and responsibilities of a hospital pharmacist, storage of medications in hospital pharmacy, and different inpatient and outpatient hospital pharmacy services. Furthermore, for training in the nutrition department, the students were given different e-cases emphasising the impact of nutrition on food-drug interactions like the interaction of grapefruit juice with statins, the effect of vitamin k-rich food on the international normalized ratio (INR), etc. for identifying potential drug-food interactions using PEPID drug interaction checker. The students were required to submit reports on their nutrition and hospital pharmacy rotation along with a drug information query. The students were also shown videos for virtual interdisciplinary rounds experience. Figure 2 summarises all the virtual hospital placement activities employed during the COVID-19 pandemic.

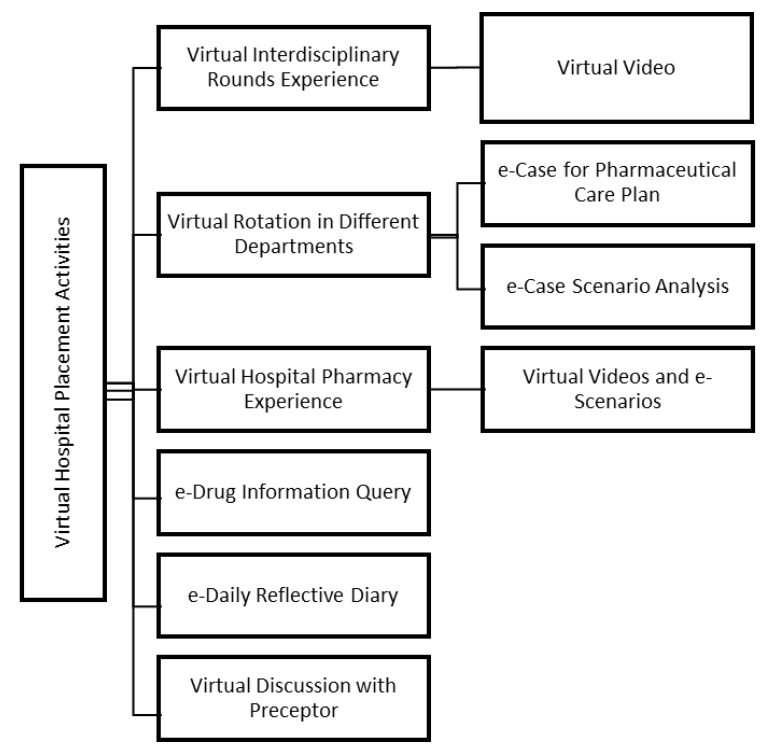

Figure 2: Virtual hospital training experience

\section{Student and preceptor feedback}

Feedback from students as well as preceptors was obtained at the end of the virtual experiential training. Students' feedback was taken by sending out an online survey as a Google form, whereas preceptors shared their feedback during the focus group discussions organised at the end of the training.

The virtual rotation student feedback survey consisted of 24 questions with 20 closed-ended questions and four open-ended questions. The closed-ended questions were related to different aspects of the experiential training like access, delivery, content, activities and usefulness. The open-ended questions like "the best part of virtual rotations was" aimed to 
obtain the qualitative feedback of the training from the students. Data from the feedback survey was analysed using descriptive statistics, and the results were presented as frequency and percentages.

Out of the total 52 final-year Bachelor of Pharmacy students, to whom the feedback survey was sent, four students did not complete the feedback survey $(92.3 \%$ response rate). Of the 48 students, the majority (79.2\%, $n=38$ ) were of the opinion that virtual rotations were useful in terms of ease of access and convenient timings. Almost all students $(89.6 \%, n=43)$ believed that the virtual rotations provided them with the real-time training experience simulating clinical settings despite the COVID-19 associated limitations. A high proportion of students $(70.8 \%, n=34)$ felt virtual rotations' mode of delivery through the different online platforms was appropriate and effective. Nearly half of the students $(50 \%, n=24)$ stated that they acquired sufficient knowledge, necessary skills, and competence during virtual placements. A majority of the respondents (89.6\%, $n=43)$ felt that e-learning through virtual rotations was challenging. It is noteworthy that a good number of students $(60.4 \%, n=29)$ agreed that virtual rotations could not completely replace onsite rotations, emphasising the importance of real clinical setting-based training. Overall, the majority of the students $(89.6 \%, n=43)$ reported that the virtual experiential training for both hospital and community pharmacy placements was informative, skill-based, and valuable.

Lack of patient encounters and face-to-face interactions, increased number of online tasks and assignments, increased screen time leading to inactivity and affecting the sleep quality, reduced socialisation, difficulty in keeping themselves motivated, were some of the challenges and drawbacks identified by the students during the online experiential training.

Keeping in view the quick and unexpected restructuring of the experiential training amid the COVID-19 restrictions, preceptor feedback was sought through focus group discussions at the end of the training. Focus group discussions were aimed at getting vital insights from the preceptors pertaining to the overall organisation and implementation of the experiential training.

The preceptors felt that the training, in general, was well conducted regardless of the challenge of quickly adapting to a virtual mode of training. All the preceptors were of the opinion that the virtual activities conducted for hospital, as well as community pharmacy rotations, were able to achieve the desired learning outcomes of the training. However, the preceptors acknowledged that all the virtual activities and assessments could be further refined and improved with detailed planning and execution in anticipation of a rerun of the same in future.

Furthermore, the preceptors recognised various challenges faced during the conduct of the training. One of the challenges, which all the preceptors highlighted, was to keep the students actively engaged in the virtual activities. Increased number of online sessions combined with novel e-documentation techniques were also identified as a challenge by the preceptors. In addition to this, technical issues related to electronic devices and internet connectivity posed formidable challenges for the preceptors.

\section{Outcomes and recommendations}

The COVID-19 pandemic exposed educators and students to an unprecedented situation that made the students' teaching and learning a challenge. Institutions across the globe grappled with the challenge of ensuring continuity of education and, in pursuit of this continuity, were forced to deliver their educational programs online (Almetwazi et al., 2020; Elnaem et al., 2020; Khan, 2020; Lyons, Christopoulos, \& Brock, 2020; Nizhenkovska, Kuznetsova, \& Narokha, 2020; Wahab \& Zainal, 2020).

Given this unforeseen COVID-19 situation, the Department of Clinical Pharmacy and Pharmacology redesigned their experiential training as a virtual experience. This virtual experience's main aim was to provide an alternative solution for the initial onsite experiential training amid the COVID-19 restrictions. Similar redesigning of the experiential training was done by a number of pharmacy schools all around the world amid the peak of COVID-19 restrictions (Almetwazi et al., 2020; Elnaem et al., 2020; Wahab \& Zainal, 2020). The virtual experiential training successfully achieved the desired learning outcomes and was well received by the students. However, there are areas for future improvement, which include increasing the duration of the experiential training, using diverse online training platforms to cover a range of practice-based activities, inclusion of more patient-centric activities and provisions for live virtual patient encounters.

Many hurdles and challenges were also experienced during the virtual training. The main challenges faced by students during the virtual sessions were maintaining active engagement and participation, technical issues, increased screen time and increased number of online tasks and assignments. Furthermore, from the preceptor's viewpoint, the virtual training was well planned and organised despite the sudden transition to the virtual mode, increased number of online sessions 
and hasty adaptability to novel e-documentation methods.

Irrespective of the successful conduct and acceptance of this virtual experiential training, some limitations need to be acknowledged. First, this was a first-time experience involving a single institution. Second, there was limited time on hands for restructuring the experiential training due to the unprecedented COVID19 situation. Third, the use of a single online platform (Google) might have restricted the spectrum of activities related to the training. Fourth, there was a limited interprofessional component in training.

Based on the authors' experience, the authors are making some recommendations, which might be beneficial for other institutions during similar situations. Firstly, efforts should be made to develop an integrated model for experiential training in the future, which can be an amalgamation of online tools and traditional experiential methods. This integrated model will enhance the onsite experiential training and will make the training future-ready. Secondly, the authors recommend the inclusion of the interprofessional component in the virtual activities and assessments. Thirdly, the authors recommend students' exposure to virtual patient encounters to make the virtual training more consequential and meaningful. Fourthly, efforts should be made to integrate artificial intelligence based technologies with experiential training. This integration will broaden the scope and will give a new dimension to the experiential training in this pandemic era. Finally, the authors strongly recommend institutions provide appropriate provisions for providing mental health support to the students during these virtual training.

\section{Ethical considerations}

Ethical clearance was taken from the RAK Medical and Health Sciences University Research and Ethics Committee.

\section{Acknowledgements}

Special thanks to Dr S. Gurumadhva Rao, President, RAK Medical and Health Sciences University.

\section{Conflict of interest}

The authors have no conflict of interest or financial disclosures to report.

\section{References}

Akel, M. El, Rahal, M., Dabbous, M., Mourad, N., Dimassi, A., \& Sakr, F. (2020). Experiential education in pharmacy curriculum: The Lebanese International University Model. Pharmacy, 9(1), 5. https://doi.org/10.3390/pharmacy9010005

Almetwazi, M., Alzoman, N., Al-Massarani, S., \& Alshamsan, A. (2020). COVID-19 impact on pharmacy education in Saudi Arabia: Challenges and opportunities. Saudi Pharmaceutical Journal, 28(11): 1431-1434. https://doi.org/10.1016/j.jsps.2020.09.008

Boyce, E. G., Harris, C. S., Bingham, A. L., Chan, E., Chapman, S. A., Chilbert, M. R., ... \& Yunker, N. S. (2020). Striving for excellence in experiential education. Journal of the American College of Clinical Pharmacy, 3(3), 678-691. https://doi.org/10.1002/jac5.1240

Christian, D. D., McCarty, D. L., \& Brown, C. L. (2020). Experiential education during the COVID-19 pandemic: A reflective process. Journal of Constructivist Psychology. https://doi.org/10.1080/10720537.2020.1813666

Elnaem, M. H., Nazar, N. I. M., \& Rahman, N. S. A. (2020). Pharmacotherapy virtual attachment during COVID-19 pandemic: Use of online experiential assessment in a Malaysian pharmacy school. Pharmacy Education, 20, 2324. https://doi.org/10.46542/pe.2020.202.2324

Hussain, F. N., Al-Mannai, R., \& Agouni, A. (2020). An emergency switch to distance learning in response to the COVID-19 pandemic: Experience from an internationally accredited undergraduate pharmacy program at Qatar University. Medical Science Educator. https://doi.org/10.1007/s40670-020-01079-9

Kallail, K. J., Shaw, P., Hughes, T., \& Berardo, B. (2020). Enriching medical student learning experiences. Journal of Medical Education and Curricular Development, 7, 238212052090216. https://doi.org/10.1177/2382120520902160

Khan, T. M. (2020). Use of social media and WhatsApp to conduct teaching activities during the COVID-19 lockdown in Pakistan. International Journal of Pharmacy Practice, 2020. https://doi.org/10.1111/iipp.12659

Lyons, K. M., Christopoulos, A., \& Brock, T. P. (2020). The COVID19 Pandemic across the academy sustainable pharmacy education in the time of COVID-19. American Journal of Pharmaceutical Education, 84(6), 8088. https://doi.org/10.5688/ajpe8468088

Nizhenkovska, I., Kuznetsova, O., \& Narokha, V. (2020). Organising distance learning for Master's in Pharmacy in Ukraine during COVID-19 quarantine. Pharmacy Education, 20, 59-60. https://doi.org/10.46542/pe.2020.202.5960

Rathbone, A. P., Rokib, T., Baqir, W., \& Campbell, D. (2019). The foundation pharmacist project: exploring new models of dual-sector postgraduate pharmacy training. International Journal of Pharmacy Practice, 27(2), 191200. https://doi.org/10.1111/ijpp.12467

Rathbun, R. C., Hester, E. K., Arnold, L. M., Chung, A. M., Dunn, S. P., Harinstein, L. M., ... \& Smilie, K. B. (2012). Importance of direct patient care in Advanced Pharmacy Practice Experiences. Pharmacotherapy: The Journal of Human Pharmacology and Drug Therapy, 32(4), e88-e97. https://doi.org/10.1002/j.1875-9114.2012.01110.x

Wahab, I., \& Zainal, Z. A. (2020). Ensuring continuity of undergraduate clinical pharmacy teaching and learning activities during crisis. Education in Medicine Journal, 12(2), 69-73. https://doi.org/10.21315/eimi2020.12.2.7 